\title{
The Status of Physical Education in Cypriot Schools
}

\author{
Diana Christodoulou
}

Faculty of Physical Education and Sport Sciences, Semmelweis University Budapest, Hungary

KEYWORDS $\quad$ physical education, educational system, education reform

\section{Introduction}

During the nineteenth century, the subject of physical education (PE) was introduced into school programs in many countries around the world. In the beginning, teachers were influenced by the military's physical training. People used to believe that physical fitness and athleticism could facilitate the achievement of moral, religious, and mental growth (Wanxiang, Zenong 2006). Physical educators wanted their students to be physically powerful; therefore their teaching methods were strict and demanding. Over the years, PE instruction has evolved according to different philosophies, particularly those of the dominant culture in the individual countries. Since 1950, there has been a gradual erosion of the frequency and quality of PE programs found in many states. PE programs disappeared from schools due to a variety of complex reasons. In some countries, physical activity and sports became an integral part of everyday life, and for that reason PE classes were neglected at schools. As sporting activity spread everywhere, the main interest of such school programs was to improve the skills and techniques in different sports. The structure of PE classes became completely different than before. In other countries, PE disappeared from curricula because of societal pressure to respond to different challenges. Learning other subjects began to be considered more important, and 
PE programs were essentially squeezed out of the priorities of public school curricula (Kientzler 2008). As a consequence the subject lost its original prestige and status.

Current research indicates that the philosophy behind PE suggests that physical education makes a significant contribution to the total education of the human being - on the basis of human physicality - by means of movement, play and sport (Haag, Haag 2003). The major purpose of PE as a subject is to promote health and encourage physical activity throughout life. It is common knowledge that PE programs in school can be identified as one of the most important vehicles for promoting health-enhancing physical activity in order to reduce the risk of heart diseases, hypertension, diabetes mellitus II and obesity (Trost 2006). Nevertheless, despite seemingly widespread acceptance, the positive impact attributed to a physically active lifestyle and the school subject itself, $\mathrm{PE}$ is drowning in deep waters. Globally speaking, PE often finds itself in a defensive position. It is frequently marginalized, suffering from "decreasing curriculum time allocation; low status esteem; budgetary controls with inadequate financial, and personnel resources" (Hardman 2003).

The aforementioned phenomena were the main interests of cross-cultural research, the findings of which were published in 2005 in a book entitled International Comparison of Physical Education, edited by Uwe Pühse and Markus Gerber. In that survey, the status of PE was compared worldwide. A total of 35 countries were involved and each author had to follow the same procedure, i.e. they had to answer the same questionnaire. The book's content is like an academic expedition meant to help readers discover the innovative and stimulating aspects encountered in the world of PE. Unfortunately, Cypriot authors were not invited to contribute to this research. Probably it was supposed that the Cypriot situation related to PE is similar to that of Greece's. The author of this paper tries to discover the PE world in Cyprus by using similar guidelines as were used by the authors of that special book. Revealing the status of PE in the Cypriot educational system and comparing it with the situation in other states could be a motivating factor for upgrading the position of PE at schools. It may also make a significant contribution to the solving of various problems found in the Cypriot educational system and stimulate those responsible for political decisions. In other words, the author of this article intends to discover the current situation and status of PE at Cypriot schools, the most dominant ideals and predominant contents of $\mathrm{PE}$, and its future as a subject in the schools.

\section{Methods}

This paper is based on comprehensive research. In the Cypriot investigation, the same concept and methods were used as in the aforementioned cross-cultural study and the results are presented according to similar dimensions. Data was collected through documentary analysis and in-depth interviews. Guidelines for the in-depth interviews $(\mathrm{N}=13)$ were adapted from the cross-cultural research. The selected participants of the in-depth interviews were Cypriot qualified PE teachers, teaching in secondary schools (10) and key-persons working in the Ministry of Education and Culture (MEC) (3).

Data used in the current study came from documentary analysis as well. In order to realize the aims of this paper the author collected information from the curricula of Cypriot public schools, with great emphasis on the PE curricula, and from relevant documents issued by the MEC.

\section{Results}

\subsection{Historical background of PE in Cyprus}

According to Doll-Tepper (2005), there have been global concerns since the 1970s regarding a perceived decline in the presence of physical education at schools. Cyprus belongs to those countries where PE as a subject has less importance today than other academic subjects at schools. In order to understand the current situation of PE in Cyprus it is essential to refer to the past. A brief historical overview serves as a means to see clearly the whole world of PE in the Cypriot society. Regarding the 
historical background of PE, it is important to mention that according to Clea (1997), there has been neither a bibliography nor any publications that refer to the probable beginning (1878) of PE at schools in Cyprus. Inevitably the Cypriot educational system was not stable in these periods because Cyprus had been occupied by various conquerors for many years. Each of them attempted to use education with the intention of promoting their political goals. They also wanted to get rid of every Greek traditional element that existed in the Cypriot educational system. Nevertheless, there is evidence that in $1892 \mathrm{PE}$ was introduced as a subject in schools.

After 1960, when the island became independent, the Cypriot educational authority sought to have their programmes approved again by the Ministry of National Education and Religion of Greece. They followed the same educational system: six classes at elementary school and six classes at the secondary general education (three classes of the gymnasiums, which were compulsory, and another three classes of the so-called lyceums). Lyceums consisted of three branches: Classiko, Practiko and Emporiko. Cypriot secondary schools adopted the Greek teaching aims and curricula. In 1976 the MEC department responsible for secondary general education created new timetables and curricula in English. Its concept was based on the previous Greek curricula. At the gymnasiums, PE classes were held 3 times per week and for the lyceums two times per week, except the Classiko branch (3 times). The lesson was taught separately for boys and girls and in primary schools the students attended PE classes twice a week.

The curriculum for primary schools was revised by the MEC in 1994. All forms of educational planning, policies, guidelines and laws remain the sole responsibility of the MEC. All public schools have to use the syllabi, curricula and textbooks prescribed, regulated and supervised by the MEC (Karagiorgi, Nicolaidou 2010). The purpose of the aforementioned curriculum was to improve teaching methods and to develop more uniform teaching material and subjects. It is surprising that since 1994 the curriculum has not been integrally changed. There were two more revised editions, in 1996 and in 2002, and three additional republications, in 2005, 2006 and in 2007.

\subsection{Time spent for $P E$ at Cypriot schools}

Time allocation for PE classes has not seen any significant change since 1994. In primary schools children attend PE classes two times per week. In the lower secondary schools three times per week and two times for the first grade of lyceums. The second and third grades of lyceums exercise only once per week. The most critical remark - crucial to be mentioned here - is that no PE curriculum has existed for the gymnasiums (lower secondary) at all. When asked about it, the answer from those responsible for it was surprising: PE teachers do not follow an official written curriculum to teach at gymnasiums, they just follow instructions and advice given by the inspectors of the PE. It can be seen also in the website of the MEC that the only available curriculum for the secondary schools is the one for lyceums (www.moec.gov.cy).

\subsection{Plans for the future}

In 2003 the educational authorities of Cyprus observed and realized that there were many severe problems and weaknesses in the education of the students. Seven academics were appointed with the purpose of reshaping the highly centralized educational system. The most significant propositions for change in their project were the following:

1. Institutional framework of governing, power and monitoring,

2. New structure of educational system and school institutions,

3. Content of education: curricula, school knowledge, and pedagogic educational process,

4. Higher public and private education,

5. Education and training of teachers,

6. Evaluation of educational work and teachers (Committee on Educational Reform 2004). 
In 2008, following the acceptance of some of the above propositions, another committee was appointed that was responsible for the formation of curricula. In turn, additional experts were appointed in 2009 that were specialists for each subject included in the curricula. Finally they succeeded in creating a new curriculum in 2010 that involves all levels of education: pre-primary, primary and secondary education. Their project comprises the content of the different subjects, teaching methods, and system of evaluation, the educational environment and the relationships between educational system, parents, students, and teachers.

In the current period (school year 2010-2011), teachers and inspectors of each subject comprised in the curricula started their training in order to use the required teaching materials and methods. In the school year 2011-2012, according to their plans, the renewed curriculum will be introduced in primary schools and gymnasiums; in 2012-2013 it will be introduced in the first grade of lyceums; in 2013-2014 in the second grade; and in 2014-2015 the third grade of lyceums. There are many changes made to the whole educational system including certain curricula, this is the reason that a longer period of time is required in order to successfully introduce it. Generally speaking, Cyprus faces numerous challenges, particularly since it became a member of the European Union in May 2004, which led to unprecedented efforts to improve the educational system (Karagiorgi, Nicolaidou 2010). It is expected that a new mentality will followed by students, teachers and parents alike, with the intention of realizing the aims of the new system. The good news is that there is no intention for the time allocation of PE classes to be reduced. One of the new approaches to PE is that not a single academic subject is inferior to others. At primary schools the time allocation of PE will be the same as before. At lower secondary schools (gymnasiums) there will be three PE classes per week for the first and second grades, and two for the third grade. In lyceums two PE classes are planned per week for all grades.

\subsection{Current status of $P E$ and its legitimacy}

Another vital issue to be considered is the current status and legitimacy of PE in the educational system. As it was mentioned in a previous section, PE class in Cypriot schools is inferior in practice to other academic subjects. As Yiallourides (1998) mentioned in his $\mathrm{PhD}$ dissertation, $\mathrm{PE}$ lessons are cancelled when time is required for reading and mathematics or for the revision and test taking in other academic subjects. Although PE is a compulsory subject within the curriculum - which officially means that it is legitimate - many Cypriots perceive it as a non-productive educational activity. They believe that PE class at schools is a waste of time and that PE is only about blowing a whistle (Hardy 1997). Apparently there are few PE teachers who teach that way, as they have to. In many instances children were complaining that the only thing that they had been doing in PE classes was kicking the ball and nothing else. A great number of qualified PE teachers have the feeling that their special role is not highly valued by society (Christodoulou 2009). However, we have to emphasize that this criticism cannot be generalized for every PE lesson or for every PE teacher. There are many PE teachers who love their job, are professionals, and try to do their best in order to transmit their knowledge related to sports and healthy active lifestyles to the students.

\subsection{Associated problems need to be resolved}

Another critical concern regarding PE teachers in Cyprus is their oversupply in comparison with the demands of the public sector in the society. Almost every newly graduated PE teacher is compelled to undertake other jobs not related to teaching. All of them are registered on an official waiting list arranged by the year of their graduation and have to wait for some years until they are offered employment at public secondary schools (Christodoulou 2010). In 2007 there were 1,488 (561 females and 927 males) qualified PE degree holders who could not teach at schools. The MEC prohibited the appointment of PE teachers in elementary schools. Traditional elementary teachers are obliged to undertake an extra course in PE in order to teach the subject themselves. In other words, 
qualified PE teachers cannot use their university knowledge and education to teach PE. Some of them are forced to work in jobs not related to sports, and others are working as coaches and/or instructors. But what will happen after fifteen to twenty years when they will be invited to work at schools? Will they be ready to teach all the sporting events and exercises with patience and love?

On the other hand, as it was mentioned above, elementary school teachers are those who teach $\mathrm{PE}$ in primary schools. It is believed that children aged six to twelve are not properly taught the subject of PE. PE teachers are obviously more familiar with the whole world of sports and also with the teaching methods of PE. The duration of their studies is three to four years and during these years they get extensive experience in education of children in different ages. How is it possible for traditional elementary teachers to teach PE at the same level, if their education in PE lasted only for a few months? Do they really know what and how to teach to children in PE classes in the same way that qualified PE teachers know? Besides, research conducted in 2002 indicates that the prevalence of obesity among Cypriot children aged 10 to 11 is around $10 \%$. This percentage shows that the occurrence of obesity in Cyprus is as high and in certain instances it is even greater than in other developed countries (Savva et al. 2002). It is well known that obesity is associated with a lack of physical exercise (which facilitates energy expenditure) and energy intake (Jabłońska et al. 2008). If children are not physically active and do not take care of their nutrition, the consequences can be tragic. Obesity and overweight may lead to illnesses and numerous health hazards (Jarosz, Rychlik 2008).

\subsection{The most dominant ideals of $P E$ and its contents}

In the primary schools (six to twelve years), the aim of PE is to promote healthy growth in students, including physical, spiritual and emotional development. Another purpose is to contribute to the improvement of their health and their smooth integration into society. The curriculum for elementary schools is divided into two circles in all three grades. The first three grades stress the development of basic skills. Certainly, teachers do not expect the children to play sport and exercise with excellent technique. The basic exercises taught include moving around in different ways, learning how to handle and control apparatus safely, learning various body positions and movements like stretching and balance, games, and relays. In the second cycle, teachers continue to teach basic kinetic elements, with the intention of teaching the more specialized skills related to sports. Students are introduced to gymnastics, track and field, ball games, and dance. As a final point, it is important to note that in the current curriculum there is no chapter or paragraph that explains the evaluation process of PE.

Curriculum for the lower secondary schools - as mentioned above - does not exist. The aims of PE in lyceums are the following:

a) to develop positive attitudes and to obliterate violence and hooliganism from sporting events,

b) to ensure equal opportunity for students concerning their participation in PE classes,

c) to encourage students to engage in physical activities throughout their lives.

The purpose of PE classes for the first grade of lyceum is to develop the students' physical abilities and skills, and to prepare them for the second and third grades. Students first of all have to improve their strength, endurance, speed and flexibility. They have to learn the different techniques and rules for each sporting event in track and field, and in some ball games. PE teachers have also to teach them two traditional Cypriot dances and many exercises in order to improve their rhythm and tempo. Finally they have to learn some theoretical knowledge that involves lectures related to nutrition, healthy lifestyle and stress control.

The evaluation process is not described in detail in the curriculum. It is only explained that it happens frequently with measurements and tests.

The purpose of PE in the second and third grades is similar: 
1. To continue learning different techniques and skills of all sports,

2. To increase or maintain the students' interest for physical activities,

3. To build basic foundations in sports, mainly fair play,

4. To emphasize the recreational aspect of PE.

The evaluation of students in PE depends on seven factors:

1. Their readiness before and after PE class (constitutes $5 \%$ of the total mark),

2. The required uniform of $\mathrm{PE}(5 \%)$,

3. Their attendance or absence from PE (10\%),

4. Their attitude and behavior towards the PE teacher, students and the subject itself (5\%),

5. Their physical abilities and skills (50\%),

6. Their knowledge in oral and written form (25\%),

7. Oral and/or practical presentation (5\%).

The evaluation process for the third year students is also not written in their curriculum. Moreover it must be emphasized that in the second and third grade of lyceums, students are able to choose some extra subjects to be specialized. PE is one of those subjects and in the curricula there are additional schedules for it with extra time in the timetables as well.

\section{Conclusions}

Schools have been identified as the institution with primary responsibility for promoting physical activity (McKenzie 2007), a fundamental factor of human development. Lack of physical activity may lead to delays - or even a deficiency - in one's overall development (Bronikowski, KrolZielinska, Tarnas 2000). This article highlights the necessity of supporting PE in the Cypriot educational system. It seems that Cypriots are aware of several benefits that can be gained by physical exercise, but they are not positive towards PE classes in the composition of the curricula.

Despite the fact that the results of "Special Eurobarometer" (2004) indicated that 97\% of Cypriot citizens believe that sport can serve as a mean of improving health, they do not consider PE class as a "real discipline" at schools. It is commonplace that regular sport and exercise help children build and maintain healthy bones, muscles and joints, controlling body weight, reducing fat and developing efficient function of heart and lungs. It should be emphasized that schools have unique opportunities to provide adequate physical activity for all children on an equal basis through official compulsory physical education programs (WHO 2003). If authorities and decision-makers share the assumption that a physically active lifestyle can make a significant contribution to individuals' quality of live, they must ensure that PE remains an important element in school (Brandl-Bredenbeck 2005).

In the European Union there have been efforts to raise the population's awareness about the importance of sport in an educational context as well as increasing the significance of physical activity in school curricula. Physical educators in Cyprus seem to experience problems in convincing authorities at the MEC about the important role of PE (Doll-Tepper 2005). PE as a subject has to be taught by professionally qualified PE teachers. They have to be able to stimulate young people to participate regularly in physical activities and provide a professional education from elementary school onwards. Unfortunately, in Cypriot elementary schools there are some traditional elementary teachers who do not love sporting activities and physical exercise, but they are obliged to teach this subject to the students. Moreover, in secondary schools there are some PE teachers, mostly too old who do not teach the subject with enthusiasm any more. On the other hand, there are more than a thousand of PE degree holders who might love and feel anxious to teach what they have learned in universities; but they are not able to get employed at schools. On the other hand there is evidences that shows that obesity in childhood has been increasing to a great extent in Cyprus. All of the aforesaid concerns are urgent problems for the Cypriot society, especially for the educational system. 
In conclusion, the good news is that finally the Committee responsible for the Educational Reform managed to prepare new curriculum based on the up-to-date necessities of the Cypriot educational system. All the school subjects are included in one special book published by the MEC. All the aims and purposes of PE are explained as well. It also refers to the teaching strategies from the pre-schooling ages until the last grade of lyceum. The evaluation process is described in detail in order to help both students and teachers perform better. The only thing that remains to obtain a better educational system is to realize in practice all the objectives formulated by that particular committee.

\section{REFERENCES}

Brandl-Bredenbeck, H. P. (2005). Comparative Physical Education -why, what and how? In U. Pühse, M. Gerber (Eds.), International comparison of physical education. Concepts - problems - prospects. Oxford UK: Meyer and Meyer Sport.

Bronikowski, M., Krol-Zielinska, M \& Tarnas, J. (2000). Motor fitness and social development in 13 year old pupils from Poland. Gymnica, 3(2), 41-49.

Christodoulou, D. (2009). Cypriot PE Teachers' Opinions on Occupational Prestige and Social Capital. Physical Culture and Sport. Studies and Research, vol. XLVI, 116-122. DOI: 10.2478/v10141-009-0009-x.

Christodoulou, D. (2010) Health awareness and sporting behaviour of qualified Cypriot physical education teachers. Biomedical Human Kinetics, 2, 54-57. DOI: 10.2478/v10101-010-0013-y.

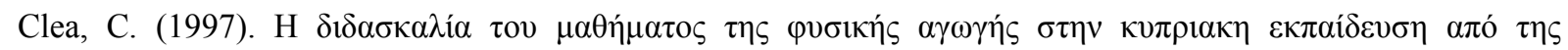

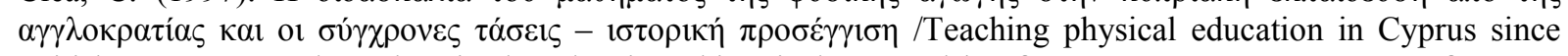

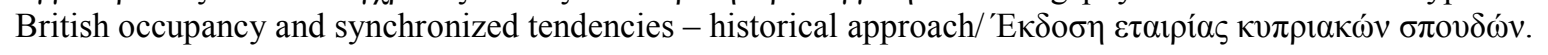

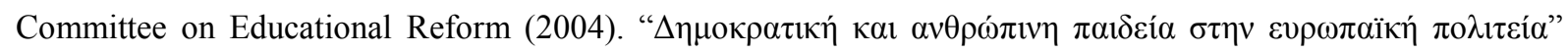
/Democratic and humanistic education in the Euro-Cyprian policies/ - http://www.paideia.org.cy/index.php

Doll-Tepper, G. (2005). Foreword In U. Pühse, M. Gerber (Eds.), International comparison of physical education. Concepts - problems - prospects. Oxford UK: Meyer and Meyer Sport.

Haag, H. \& Haag, G. (2003). Dictionary on sport, physical education and sport science. Kiel: Institut für Sport und Sportwissenschaften.

Hardman, K. (2003). Worldwide survey on the state and status of physical education at schools: foundations for deconstruction and reconstruction of physical education (pp. 15-34). Schorndorf: Hofmann.

Hardy, C. (1997). Sources of conflict during the school experience of pre-service physical education teachers. European Physical Education Review, 3(2), 116-128.

Jarosz, M. \& Rychlik, E. (2008). Overweight and obesity among adults in Poland, 1983-2005. Advances in Medical Sciences, 53(2), 158-166.

Jabłońska, J., Raganowski, P., Pyra, K., Jurkiewicz, J. \& Mandryk, M. (2008). Obesity as a problem of contemporary medicine. Annales Universitatis Maria Curie - Skłodowska, vol. XXI (1), 23.

Karagiorgi, Y. \& Nicolaidou, M. (2010). Opening Pandora's box: school autonomy in Cyprus and emerging implications for school leaders. Management in Education, 24(2), 62-68.

Kientzler, L. A. (2008). Wellness: health, nutrition and physical education. $21^{\text {st }}$ Century Education: A Reference Handbook. SAGE publications - online $<\mathrm{http}: / /$ www.sage-ereference.com/education/Article_n41.html $>$.

McKenzie, T. L. (2007). The preparation of physical educators: a public health perspective. Quest, 59(4), 346357.

Pühse, U. \& Gerber, M. (2005). International comparison of physical education. Concepts - problems prospects. Meyer and Meyer Sport. Oxford UK.

Savva, S. C., Kourides, Y., Tornaritis, M., Epiphaniou-Savva, M., Chadjigeorgiou, C. \& Kafatos, A. (2002). Obesity in children and adolescents in Cyprus - prevalence and predisposing factors. International Journal of Obesity, 26, 1036-1045.

Special Eurobarometer (2004). The citizens of the European Union and sport. Summary. European Commission.

Trost, S. (2006). Public health and physical education. Handbook of Physical Education. SAGE publications online $<$ http://www.sage-ereference.com/hdbk_physed/Article_n10.html $>$.

Wanxiang Y. \& Zenong, Y. (2006). Physical education. Encyclopedia of Educational Leadership and Administration. SAGE publications - online $<\mathrm{http}: / /$ www.sage-ereference.com/edleadership/Article_n434.html $>$.

WHO (2003). Health and development through physical activity and sport. Geneva. 
Yiallourides, G. (1998). Factors influencing the attitudes of 9-14 year old Cypriot pupils towards physical education. Unpublished $\mathrm{PhD}$ thesis, University of Manchester.

www. moec.gov.cy (Curricula).

AUTHOR'S ADDRESS: Diana Christodoulou

Ayiou Andreou st. 95

1040 Nicosia, Cyprus

Email: diana_chr@hotmail.com 